\title{
Personal determinants of mental reliability of an athlete
}

\author{
Kolosov A.B. ${ }^{1 \mathrm{ABDE}}$, Volianiuk N.Yu. ${ }^{2 \mathrm{ABCDE}}$, Lozhkin G.V. ${ }^{3 \mathrm{ABDE}}$, Buniak N.A. ${ }^{4 \mathrm{ADE}}$, Osodlo V.I. ${ }^{\text {SACDE }}$ \\ ${ }^{\prime}$ Laboratory of Current and Operative Control of National Teams' Performance, State Scientific Research Institute of \\ Physical Culture and Sports, Ukraine \\ ${ }^{2,3}$ Department of Psychology and Pedagogy, National Technical University of Ukraine «Igor Sikorsky Kyiv \\ Polytechnic Institute», Ukraine \\ ${ }^{4}$ Department of Psychology in production Ternopil Ivan Puluj National Technical University, Ukraine \\ ${ }^{5}$ Social Studies Institute, National Defence University of Ukraine named after Ivan Cherniakhovskyi, Ukraine
}

Authors' Contribution: A - Study design; B - Data collection; C - Statistical analysis; D - Manuscript Preparation; E - Funds Collection.

\begin{abstract}
Purpose: $\quad$ Stability of performances at competitions with a preset effectiveness in the presence of sports competition is the result of the reliable functioning of the psyche of an athlete. The hypothesis of the study - mental reliability is associated with certain individual psychological properties, the similarity and difference of which is determined by the level of success of an athlete.

The purpose of the study is to identify a set of personality determinants that affect the mental reliability of an athlete.

Material: $\quad$ The study involved 58 fencers aged $17-18$ years $(M=17.47 \mathrm{SD}=0.53)$. In this paper, the measurement of the mental reliability of an athlete was carried out using an integral assessment of the success of sports activities developed by E.V. Melnik and E.V. Silich. According to the final success rate, the total sample of the subjects was divided into 2 groups: «successful» and «unsuccessful». For the study of the leading individual psychological properties of the personality, 16-PF multifactor personality questionnaire by R.Kettel was used in the work. The data processing package SPSS was used for data processing.

Results: $\quad$ Significant differences were established between the groups of «successful» and «unsuccessful» athletes in the majority of individual psychological properties. The importance of focused analysis and the development of individual mental properties as internal prerequisites for the mental reliability of fencers has been acknowledged. The relationship of personal factors with the success in sports activities is presented. A high level of correlation between the integral indicator of the success of competitive activity and intelligence (factor B), emotional stability (factor C), emotional hardness (I), confidence (O), independence and autonomy (Q2) is revealed. A significant correlation was found between the average level of success in sports and caution (F). This confirms the possibility of applying the methods of research of individual psychological properties of a person when studying the causes of lesions and the prerequisites for the erroneous actions of an athlete.

Conclusions: $\quad$ The success of sports activities of fencers does not depend on one individual psychological property of a person. This is the result of a combination of most of them. A greater number of reliable relationships have been revealed between the final indicator of the fencers' success in sports activities and personal factors from the emotional properties group as compared to communicative and intellectual properties groups.

Keywords: athlete, mental reliability, success, personal properties, determination.
\end{abstract}

\section{Introduction}

Rapidly evolving, the problem of a mental reliability in a short time has become leading in the sport of higher achievements. The authors of works on the theory of reliability indicate a number of objective reasons for the actualization of this problem $[1,2]$. First of all it is increasing requirements for training and personal characteristics as well as moral and ethical qualities of highly skilled athletes. Another reason is associated with the expansion of the geography of international competitions and the steady increase in the number of applicants for the highest awards. The third reason is the commercialization of sports, which is associated with the achievement of the highest possible result in competitions and obtaining high incomes. Competitive activity is associated with many different «knock-down» factors, such as: internal and external uncertainty of the outcome

\footnotetext{
(C) Kolosov A.B., Volianiuk N.Yu., Lozhkin G.V., Buniak N.A., Osodlo V.I., 2019
}

doi:10.15561/18189172.2019.0205 of the fight, fatigue, the actions of the audience, the bias of refereeing, etc. Stability of performances at competitions with a preset effectiveness in the presence of sports competition is the result of the reliable functioning of various subsystems of the body and psyche of an athlete.

Reliability - is a term borrowed from the technical sphere due to the steady increase in the complexity of technical means. The concept of reliability is associated with efficiency, trustability, infallibility and stability [3]. Psychological reliability can be defined as a complex internal property that allows athletes to consistently and effectively perform at competitions for a certain time.

The effectiveness of ensuring the mental reliability of an athlete depends on what level of knowledge about it we have. There are four main levels of the mental reliability study of a qualified athlete: biological, biomechanical, psychological, and socio-pedagogical.

The biological level of reliability of an athlete includes the functioning of the cardiovascular, respiratory, muscular, central nervous system and its analyzers. 
Biological reliability is understood as a level of regulation and a ratio of elements of the process itself, when its optimal course is provided with reserve capabilities, with interchangeability of links, with a quick return to the initial state, with sufficient lability or plasticity guaranteeing adaptation and restructuring according to the concept proposed by Markosyan [4]. A trained and experienced organism possesses not only a wide range of regenerative adaptations, but also the ability to use its reserves and biological reliability more efficiently [5-7].

Biomechanical level of reliability is associated with the technical training of an athlete. The method of performing a sporting action must be expedient, rational, effective and focused on the individual biomechanical features of the athlete. Modern computer tools allow you to create reference models of sports equipment, thereby ensuring the possibility of assessing its effectiveness and testing in a variety of conditions.

The psychological level of reliability provides an integrated role, creating conditions for the realization of biological, biomechanical and socio-pedagogical reliability. The most important features of psychological reliability include: constancy of perception, concentration of attention, emotional stability, self-regulation, noise immunity, volitional qualities, motives of activity and their hierarchy in a person [8].

The socio-pedagogical level of reliability includes a wide range of problems in the formation of an athlete's personality in the context of cooperation and teamwork. The reliability of teamwork is a relatively little developed field of sports psychology.

A fuller account of the personality characteristics of an athlete, the characteristics of his motives as well as character and temperament traits open new horizons not only for the psychological analysis of erroneous actions, but also for taking measures to prevent them.

Achieving a high level of mental reliability creates a margin of competitive action, provides a wide range of adequate reactions of an athlete in the most unexpected competitive situations.

Experience in studying the role of personal and social factors in ensuring reliability has been accumulated in research into the problem of activity in extreme conditions.

So, when studying the relationship between the personal characteristics of flight school cadets and learning success, obtained using the Eysenck Personal Questionnaire (EPI), it was found that $60 \%$ of neurotic introverts, $36 \%$ of stable extroverts and only $14 \%$ of stable introverts have unsatisfactory marks. Thus, it was found that, compared with extroverts - introvert cadets better assimilate various flight training disciplines and practical exercises. Similar results were obtained by Reinhardt on a sample of US pilots, as well as during a survey of British military pilots [9].

A broad overview of the research on the role of personality traits, cognitive processes, mental properties and states in the formation of a predisposition to violations of professional reliability is given in the work of Kotik and Emelyanov [10]. The most important personality traits for the profession of the pilot are emotional stability, firmness of convictions, and self-confidence. Studies of Dikaia [11] revealed that personality traits such as aggressiveness, tendency to lead, rigidity and self-control reduce the possibilities of self-regulation and determine the state of stress. A number of personality traits have been studied that predispose to inadequate and chaotic behaviour under extreme conditions. These include emotional instability, inadequate optimism, and deviant social behaviour [5].

The conditions of wrestling in situational sports (sports games, martial arts), cause a person in a heightened neuropsychic tension according to the results of research by a number of authors such as: Osipov et al., Doroshenko et al. The authors came to an important conclusion about the need to take into account the personal, subjective factor in the process of regulating the intensity of the load. It is not right to underestimate human potentials to regulate load's intensity, basing on subjective feelings. [12, 13].

Significant perceptual and intellectual indicators of the psycho-physiological functions of female volleyball players are determined in motor tasks that are complex and manifest themselves in conditions unusual for players as it was determined in studies of Kozina et al. [14].

According to previous studies, Laborde et al. [15]; Madzar et al. [16]; Malinauskas et al. [17]; Znazenet al. [18] it is shown that elite players (professional athletes) use various types of mental skills aimed at controlling competitive anxiety and regulating their behaviour.

However, it should be noted that the established laws on the relationship of mental reliability and personal qualities are mainly related to specialists of extreme activities, as well as athletes playing sports. Unfortunately, the available reference materials lack systematic knowledge about the interrelationship of these complex phenomena among fencers.

The hypothesis of the study - mental reliability is associated with certain individual psychological properties, the similarity and difference of which is determined by the level of success of an athlete.

The purpose of the study is to identify a set of personal determinants that affect the mental reliability of an athlete.

\section{Material and methods.}

\section{Participants.}

The study involved 58 fencers aged 17-18 years (M $=17.47 \mathrm{SD}=0.53$ ), who underwent a comprehensive examination in the laboratory of current and operational control of the preparation of national teams of the State Research Institute of Physical Culture and Sports.

\section{Research Design.}

The integral assessment of the success of sports activities was carried out with the help of methods developed by Melnik and Silich [19] for expert evaluation, which consists of 25 indicators that reflect the subjective and objective aspects of the activity. The structural components of the success of sports activities were recorded in the protocol, in which individual features were evaluated on a 10-point scale. External objective assessment consisted of 9 indicators: sports category, rank; 
sports experience; the best place according to the rank of the competition; rating in the federation; perspectivity (sporting status) - from a candidate for the national team core; the number of competitions per year; the highest rank of the competition and the occupied place. The internal (subjective) assessment of the athlete's sporting success consisted of 15 indicators. The latent assessment of success was an integral indicator, also expressed on a 10-point scale, which was calculated based on the judges' ratings.

According to the final success rate, the total sample of subjects was divided into 2 groups: «successful» - $\mathrm{n}=19$ (average value of the final success rate of sports activities - 21.47; SD = 1.46) and «unsuccessful» - $\mathrm{n}=17$ athletes (average value of the final success rate -10.88 ; $\mathrm{SD}=$ 1.61). 22 athletes from the total sample were ranked as moderately successful in terms of the final success rate of sports activities, therefore their results were not taken into account in the process of further analysis.

In the present study, the 16-PF multifactorial personality questionnaire was used to study the leading individual psychological properties of the personality. A personality consists of stable interrelated elements (properties, traits) that determine its inner essence and behavior according to this theory [20]. The questionnaire diagnoses the personality traits, which R. Cattell called constitutional factors. Examination and treatment procedures are standard. As a result primary data was obtained.

\section{Statistical Analysis.}

The research results are processed by the methods of mathematical statistics. These methods are the calculation of the average data, standard deviation and correlation analysis. When comparing the two empirical distributions, the Pearson criterion, the Kolmogorov-
Smirnov criterion, and the Student's t-criterion were used. The data processing package SPSS 17.0. was used for data processing.

\section{Results.}

Table 1 presents the results of the study of the individual psychological properties of the subjects, who are in the group of «successful» and «unsuccessful» athletes. The table shows the average sample values for personality factors and Student's t-criterion values $(\mathrm{T})$.

Let's analyze the differences of personality traits of successful and unsuccessful athletes using the Student's $\mathrm{t}$-criterion. Significant differences in factor B $(\mathrm{t}=7.089$, $p=0.000)$ are revealed. Successful athletes are more erudite and quick-witted, able to act in original and unusual ways in various situations. Differences in factor $\mathrm{C}$ are found $(\mathrm{t}=11.893, \mathrm{p}=0.000)$, which determines the strength of their nervous system, since the severity of factor $\mathrm{C}$ depends on innate premises, is a central element of the ability to tolerate frustration and correlates with the concepts of a strong and weak nervous system by Pavlov [21]. High scores on factor C (6.26) indicate such features of successful athletes as emotional stability, emotional maturity, adequacy of explanations and realistic expressions of their emotions and moods.

Qualities such as perseverance, aggressiveness, conflict and the desire for self-affirmation are less pronounced among successful athletes compared to unsuccessful, which confirm the significance of difference in factor $\mathrm{E}(\mathrm{t}=-6.949, \mathrm{p}=0.000)$. Great responsibility, sobriety and a serious attitude towards life are confirmed by the difference in the $\mathrm{F}$ factor $(\mathrm{t}=-19.835, \mathrm{p}=0.000)$. Factor $\mathrm{F}$ characterizes a stable property, not a state of a person. It depends on heredity, family traditions, as well as cultural norms and living conditions. Low scores on

Table 1. Statistically significant differences in the level of development of personal qualities in a group of successful and unsuccessful athletes

\begin{tabular}{lllll}
\hline Factor & «Successful» group & «Unsuccessful» group & \multicolumn{2}{l}{ Differences } \\
& Mean \pm Std. Deviation & Mean \pm Std. Deviation & t & p \\
\hline A Warmth & $4.94 \pm 1.12$ & $5.00 \pm 0.86$ & -0.203 & 0.841 \\
B Reasoning & $5.36 \pm 0.89$ & $3.65 \pm 0.99$ & 7.089 & $\mathbf{0 . 0 0 0}$ \\
C Emotional Stability & $6.26 \pm 1.04$ & $3.41 \pm 0.75$ & 11.893 & $\mathbf{0 . 0 0 0}$ \\
E Dominance & $4.89 \pm 0.89$ & $6.29 \pm 0.98$ & -6.949 & $\mathbf{0 . 0 0 0}$ \\
F Liveliness & $3.73 \pm 0.65$ & $6.71 \pm 1.16$ & -19.835 & $\mathbf{0 . 0 0 0}$ \\
G Rule-Consciousness & $5.21 \pm 0.78$ & $5.35 \pm 0.93$ & -0.772 & 0.450 \\
H Social Boldness & $5.52 \pm 1.02$ & $6.82 \pm 0.95$ & -5.527 & $\mathbf{0 . 0 0 0}$ \\
I Sensitivity & $3.57 \pm 0.76$ & $6.71 \pm 1.04$ & -17.758 & $\mathbf{0 . 0 0 0}$ \\
L Vigilance & $3.94 \pm 0.85$ & $5.65 \pm 0.86$ & -8.751 & $\mathbf{0 . 0 0 0}$ \\
M Abstractness & $5.47 \pm 0.84$ & $5.12 \pm 0.85$ & 1.833 & 0.083 \\
N Privateness & $5.68 \pm 0.94$ & $5.53 \pm 0.94$ & 0.711 & 0.486 \\
O Apprehension & $3.89 \pm 0.93$ & $6.47 \pm 1.06$ & -11.985 & $\mathbf{0 . 0 0 0}$ \\
Q1 Openness to Change & $5.42 \pm 1.01$ & $5.18 \pm 0.88$ & 1.033 & 0.315 \\
Q2 Self-Reliance & $4.15 \pm 1.02$ & $5.24 \pm 0.97$ & -4.649 & $\mathbf{0 . 0 0 0}$ \\
Q3 Perfectionism & $6.94 \pm 0.96$ & $4.12 \pm 0.73$ & 10.844 & 0.000 \\
Q4 Tension & $4.15 \pm 0.89$ & $6.41 \pm 1.17$ & $-10,928$ & 0.000 \\
\hline
\end{tabular}


factor $\mathrm{F}$ for successful athletes suggest that strict and stringent requirements in the family contributed to their endurance, reliability, caution, and adaptability.

According to $\mathrm{R}$. Cattell, factor $\mathrm{H}$ is associated with the sensitivity of the autonomic nervous system to the threat. Persons with low scores on factor $\mathrm{H}$ have a hypersensitive sympathetic nervous system from birth, which makes them «particularly responsive to the threat». High scores for this factor indicate a functional immunity to the threat [20]. Successful athletes have a slightly lower score compared to unsuccessful ones on the factor $\mathrm{H}(\mathrm{t}=$ $5.527, \mathrm{p}=0.000)$, which suggests that they are less prone to risk and thrill.

Masculinity, stamina, practicality in deeds and realism in assessments are characteristics of successful athletes, as evidenced by the difference in factor $I(t=-17.758, p$ $=0.000)$.

Significant differences in the factor $\mathrm{L}(\mathrm{t}=-8.751$, $\mathrm{p}=0.000)$ are present. Successful athletes are more optimistic, tolerant, non-envious, compliant, and frank. A low figure for the L factor (3.94) suggests a realistic selfassessment of successful athletes.

Differences in the factor $\mathrm{O}(\mathrm{t}=-11.985, \mathrm{p}=0.000)$ are significant as well. Successful athletes are characterized by a low score on the factor O-, which indicates their faith in themselves, the ability to easily survive the setbacks and last but no least a low sensitivity to the estimates of others. The need for support and approval (factor Q2; $t$ $=-4.649, \mathrm{p}=0.000)$ is more experienced by successful athletes. Significant differences were found in the Q3 factor $(\mathrm{t}=10.844, \mathrm{p}=0.000)$. This testifies to the fact that successful athletes have a clear idea of their own «ideal», which they strive to achieve. Also this factor shows that organization and the ability to control emotions well are inherent for successful athletes. High marks (6.94) on factor Q3 indicate good social fitness of successful athletes and their ability to control their behaviour in relation to social standards. Q4 characterizes the level of excitation and voltage, expresses poorly controlled sensitivity, i.e. the level of internal restrained energy. Significant differences were revealed by the factor Q4 $(\mathrm{t}=-10.928, \mathrm{p}=0.000)$. The score on factor $\mathrm{Q} 4(4.15)$ confirms the emotional stability of successful athletes.

Let's demonstrate the characteristic of the individual psychological properties of athletes who are unsuccessful. Significant differences in factor $\mathrm{A}(\mathrm{t}=-0.203, \mathrm{p}=0.841)$, which is in the group of communicative properties, were not revealed. The average values of factor A (4.94-5.00) allow to characterize athletes of both groups as those who do not avoid relationships with people, but their own activity in establishing and maintaining contacts is low. A low score in factor B (3.65) indicates an excessive concreteness of thinking, not a high level of a verbal culture and erudition. It should be noted that the factor B+ does not determine the level of intelligence; however it is focused on the measurement of the speed of thinking. A low score for factor $\mathrm{C}$ (3.41) indicates that unsuccessful athletes have an unregulated emotionality, an acute reaction to failure, an unstable mood. Externally it is manifested as a lack of responsibility and capriciousness. Unsuccessful athletes are more domineering, independent, self-confident, stubborn, capricious, boastful and arrogant, which is confirmed by high scores on factor $\mathrm{E}$ (6.29). Unsuccessful athletes find some carelessness, as evidenced by the assessment of factor $F$ (6.71). Most likely, they are very gullible, cheerful, they are sociable and friendly. Factor $\mathrm{G}$ is an analogy of the psychoanalytic «Super-ego»: it emphasizes moral principles and has a tendency to control the «Ego» and limit the «Id» [21]. Significant differences in factor $G$ from successful and unsuccessful athletes have been identified. Scores in the range (5.21-5.35) for this factor indicate that athletes of both groups tend to comply with moral requirements and not to violate the rules, and show accuracy in business.

High scores for factor $\mathrm{H}$ (6.82) indicate stress resistance, courage, and decisiveness. But at the same time, it can be assumed that unsuccessful athletes are not always attentive to details, tend to make decisions quickly. High scores on factor I (6.71) indicate that unsuccessful athletes are sentimental, sensitive and vulnerable, often act by intuition, and are gentle towards themselves and others. Estimation according to the factor L (5.65) allows us to speak about an overestimated self-esteem, fixing on failures, and critical attitude towards the joys of other athletes. No significant differences were found in both groups of athletes in factor M. Scores by a factor in the range (5.47-5.12) indicate the originality of their worldview and the peculiarity of their behaviour, as well as the ability to act in an original and unusual way in various situations. There were no significant differences between successful and unsuccessful athletes in factor N. Athletes of both groups are well-versed in social situations, insightful about the motives and actions of other people and are able to slyly and deliberately build their behaviour, prone to intrigue. High scores for factor $\mathrm{O}$ (6.45) indicate that unsuccessful athletes are characterized by anxiety, concern, vulnerability, impressionability, sensitivity to comments and censures.

Statistically significant differences in the Q1 factor in both groups of subjects were not established. The factor Q1 defines the desire for the new as a property of thinking. R. Cattell believed that this characteristic is noticeable only when a person expresses his point of view. In the behavioural picture, a person with a low index is characterized as a "conservative» by this factor, with a high index as a radical. The range of marks (5.42-5.18) suggests that successful and unsuccessful athletes tend to be well-informed and to have critical thinking. They are tolerant to contradictions. The factor Q2 determines how conformal the personality is (dependent on the group). Significant differences in the factor Q2 indicate some dependence and attachment to a group that is distinctive for unsuccessful athletes. The factor Q3 measures the level of internal control of behaviour and characterizes the individual degree of awareness of the social requirements. The low value of factor C (3.41) and factor Q3 (4.12) reveals emotional instability and not high self-control. The factor Q4 characterizes the level of excitement and 
tension, expresses poorly controlled sensitivity. Scores by factor Q4 (6.41) indicate the presence of a large number of needs that have not been satisfied (motivation frustration), and also restlessness and impatience of unsuccessful athletes. Thus, between the groups of successful and unsuccessful athletes there are significant differences in a large number of factors.

Analysis of the differences established by the means of Student's t-criterion showed that the specific individual psychological properties of successful athletes are: erudition, ingenuity, emotional stability, restraint, responsibility, courage and resilience, practicality, insensitivity to the assessments of others, organization, good control of their emotions and behavior. It was revealed that such features as: low erudition, sharp response to failures, self-confidence, stubbornness, willfulness, trustfulness, high self-esteem, anxiety, concern, vulnerability, impressionability, dependence and group affection, emotional instability are characteristics of unsuccessful athletes.

Table 2 represents the correlation coefficients between the total success rate and the factors of the personal questionnaire by R. Cattell.

Table 2. Correlation coefficients between the final indicator of the success of sports activity and the factors of the personal questionnaire by R. Cattell

\begin{tabular}{ll}
\hline Factor & «Successful» group \\
\hline A Warmth & 0.318 \\
B Reasoning & $0.791^{* *}$ \\
C Emotional Stability & $0.711^{* *}$ \\
E Dominance & 0.084 \\
F Liveliness & $-0.558^{*}$ \\
G Rule-Consciousness & 0.294 \\
H Social Boldness & 0.084 \\
I Sensitivity & $-0.750^{* *}$ \\
L Vigilance & -0.113 \\
M Abstractness & 0.033 \\
N Privateness & 0.154 \\
O Apprehension & $-0.811^{* *}$ \\
Q1 Openness to Change & -0.029 \\
Q2 Self-Reliance & $0.694^{* *}$ \\
Q3 Perfectionism & 0.054 \\
Q4 Tension & 0.024 \\
\hline
\end{tabular}

Note: ${ }^{*}$ - the relationship is statistically significant at $\mathrm{p} \leq 0.05 ; * *$ - at $\mathrm{p} \leq 0.01$.

When interpreting the obtained results of the correlation analysis, it is advisable to use not only the severity of individual factors, but also their combinations that form complexes of communicative, intellectual, emotional and regulatory personal properties. As can be seen, the data from Table 2, factor (B), a member of the group of intellectual properties has a significant impact on the success of sports activities. This suggests that the success of sports activities depends on the intellectual capabilities of the fencer. The higher the level of generalization, the creative detailed elaboration of ideas, both own and those put forward by the coach, the higher the final indicator of the success in competitive activities. Factor (C) emotional stability positively correlates with the final indicator of the success in competitive activities $(r=0.711$ with $\mathrm{P} \leq 0.01)$. The ability to manage your emotions and mood positively affects the mental reliability of an athlete. An inverse relationship was found between the total success rate and the factors $F(r=-0.558$ with $P \leq 0.05), I(r=-0.750$ with $\mathrm{P} \leq 0.01)$ and $\mathrm{O}(\mathrm{r}=-0.811$ with $\mathrm{P} \leq 0.01)$. Caution and reasonableness (F-), realistic judgments and practicality (I-), faith in yourself, a calm attitude to the evaluations of others, courage, activity, the ability to easily experience failures (O-), all of the mentioned above determines the success of sports activities of fencers. The factor (Q2), which belongs to the group of communicative properties, also indicates the success of the sporting activity of fencers. The higher the independence in making decisions and actions as well as independence from the group, the higher the final indicator of the success of sports activities.

\section{Discussion.}

The main purpose of this study was to identify the summation of personality determinants that affect the mental reliability of an athlete. The results of the study confirmed our hypothesis that the psychological reliability of an athlete is associated with certain individual psychological properties, the similarity and difference of which is determined by the level of success of an athlete. The most difficult issue in conducting an experimental study of the mental reliability of athletes is the question of its measurement. In this paper, the measurement of the mental reliability of an athlete was carried out using the expert review methodology, which consists of 25 indicators reflecting the subjective and objective aspects of a competitive activity. According to the final success rate, the total sample of the subjects was divided into 2 groups: «successful» and «unsuccessful».

Results of our research permitted to find significant differences in the majority of individual psychological properties between the groups of «successful» and «unsuccessful» athletes. Successful athletes are more erudite and quick-witted, able to act in original and unusual ways in various situations. This fact is explained by structural changes in the brains of athletes [22]. The author points out that high-level athletes have more thickness of the cerebral cortex in the areas responsible for perception of movement and socially significant stimuli. High scores for factor $\mathrm{C}$ indicate such characteristics of successful athletes as emotional stability and emotional maturity. Successful athletes are less pronounced to obstinacy, aggressiveness, conflictedness and the desire for self-affirmation compared with unsuccessful ones. Great responsibility, sobriety and a serious attitude towards life are confirmed by the difference in the factor F. Factor $\mathrm{H}$ high scores indicate functional immunity 
to the threat. Masculinity, perseverance, practicality in business and realism in assessments are characteristics of successful athletes, as evidenced by the difference in the factor I. Successful athletes are more optimistic, tolerant, non-envious, compliant and frank. The low factor L scores suggest a realistic self-esteem for successful athletes. Low scores on the $\mathrm{O}$-factor indicate their faith in themselves, the ability to easily experience failures and low sensitivity to the estimates by others. The data we receive are in agreement with the ideas and experimental studies of other authors [23, 24]. In their studies they found that the avoidance motivation compensates for excessive stress and acts as a regulator of the athlete's psycho-physiological state. Results on the factor Q3 indicate a good social fitness of successful athletes and the ability to control their behaviour in accordance to the social standards. The score on the factor Q4 confirms the emotional stability of successful athletes.

Study of correlations between the researched indicators is also rather important. According to the results of the correlation analysis between the final indicator of the success of competitive activity and the factors of the personal questionnaire of R. Cattell in the group of successful athletes, six dependencies were found, three of which are negative.

This study revealed a set of individual psychological properties that affect the mental reliability of an athlete. It has been established that emotional properties act as leading determinants of the success of fencing sports activities. Apparently, this is due to their psychological essence, which is expressed in the activation of the central link of the analyzer and its sub-cortical structures, evaluating the usefulness or danger of incoming information. Psychologists have long been interested in the problem of the relationship between the intellectual and emotional spheres of man. L.S. Vygotsky noted that all changes in the cognitive activity of the individual are associated with profound changes in his emotional sphere [25]. The relationship between affect and intelligence was also indicated by Zeigarnik, emphasizing that thinking does not exist separately from the motives, aspirations and feelings of a person [26].

These data were obtained for the first time in fencing and are new in comparison with the data of other authors.

\section{Conclusions.}

It was established that between the groups of «successful» and «unsuccessful» athletes there were significant differences in the majority of individual psychological properties. A significant influence on the success of sports activities of fencers is exerted by a factor (B), a member of the group of intellectual properties, four factors that are included in the group of emotional personality traits: emotional stability $(\mathrm{C})$, caution $(\mathrm{F})$, emotional hardness $(\mathrm{I})$, confidence $(\mathrm{O})$ and factor $(\mathrm{Q} 2)$, included in the group of communicative properties. The success of sports activities of fencers does not depend on one individual psychological property of a person. This is the result of a combination of most of them. A greater number of reliable relationships have been revealed between the final indicator of the fencers' success in sports activities and personal factors from the emotional properties group as compared to communicative and intellectual properties groups. Periodical control and development of the emotional and intellectual properties of an athlete's personality will contribute to the growth of his mental reliability.

\section{Conflict of interests.}

The authors declare that there is no conflict of interests.

\section{References}

1. Danilina LN, Plakhtienko VA. The problem of mental reliability in sports. Moscow; 1980. (in Russian)

2. Kalandiia AT. Psychological aspects of the reliability of athletes in preparation for the Olympic Games. Vestnik Universiteta Rossijskoj akademii obrazovaniia, 2010; 1:112118. (in Russian)

3. Nikiforov GS. Reliability of professional activity. St. Petersburg: University of St. Petersburg; 1996. (in Russian)

4. Markosian AA. Reliability of the physiological system and ontogeny. In: Molecular and functional bases of ontogenesis. Moscow; 1970. P. 100-105. (in Russian)

5. Bodrov VA. Psychology of professional activity: theoretical and applied problems. Moscow: RAN; 2006. (in Russian)

6. Jagiello W, Jagiello M, Kalina RM, Barczynski BJ, Litwiniuk A, Klimczak J. Properties of body composition of female representatives of the Polish national fencing team - the sabre event. Biology of Sport. 2017;34(4):401-406. https://doi.org/10.5114/biolsport.2017.70526

7. Volodchenko OA, Podrigalo LV, Iermakov SS, Zychowska MT, Jagiello W. The Usefulness of Performing Biochemical Tests in the Saliva of Kickboxing Athletes in the Dynamic of Training. Biomed Research International. 2019. https://doi.org/10.1155/2019/2014347
8. Lozhkin GV, Soltyk AA. The research strategy of professional reliability of the subject of sports and educational activities. In: Materials of the IXInternational Conference «Rudikovskie chteniia», Moscow, May 27-28, 2013, Moscow; 2013. P.2426. (in Russian)

9. Jessup G, Jessup H. Validity of Eysenck's Personality Inventory in pilot selection. Occupational Psychology, 1971; 45: 11-23.

10.Kotik MA, Emel'ianov AM. The nature of human operator errors. Moscow: IMBP; 1975. (in Russian)

11.Dikaia LG. Mental self-regulation of the functional state of a person. Moscow: RAN; 2003. (in Russian)

12.Osipov AY, Kudryavtsev MD, Iermakov SS, Jagiello W. Criteria for effective sports selection in judo schools - on example of sportsmanship's progress of young judo athletes in Russian Federation. Archives of Budo. 2017;13:179-186.

13.Doroshenko EY, Svatyev AV, Iermakov SS, Jagiello W. The use of cardio training facilities in training 7-9-year-old judo athletes. Archives of Budo Science of Martial Arts and Extreme Sports. 2017;13:165-172.

14.Kozina Z, Sobko I, Ulaeva L, Safronov D, Boichuk Y, Polianskyi A, et al. The impact of fitness aerobics on the special performance and recovery processes of boys and girls 16-17 years old engaged in volleyball. International 
Journal of Applied Exercise Physiology. 2019;8(1):98-113. https://doi.org/10.30472/ijaep.v8i1.306

15.Laborde S, Dosseville F, Allen MS. Emotional intelligence in sport and exercise: A systematic review. Scandinavian Journal of Medicine \& Science in Sports, 2015; 26(8): 862-874. https://doi.org/10.1111/sms. 12510

16. Madzar T, Milosevic M, Hrabac P, Heningsberg N. Psychological aspects of sports injuries among male professional soccer players in Croatia. Kinesiology. 2017; 49(1):84-91.

17.Malinauskas R, Malinauskiene V. The mediation effect of Perceived Social Support and Perceived Stress on the relationship between Emotional Intelligence and Psychological Wellbeing in Male Athletes. Journal of Human Kinetics. 2018;65(1):291-303. https://doi.org/10.2478/hukin-2018-0017

18.Znazen H, Slimani M, Miarka B, Butovskaya M, Siala H, Messaoud T, et al. Mental skills comparison between elite sprint and endurance track and field runners according to their genetic polymorphism: a pilot study. Journal of Sports Medicine and Physical Fitness. 2017; 57(9):1217-1226. https://doi.org/10.23736/s0022-4707.16.06441-0

19.Mel'nik EV, Silich EV. Expert assessment of the success of sports activities (on the example of coordination sports). Sportivnaia pressa, 2010; 3:73-79. (in Russian)
20.Heather EP, Cattell and Alan D. Mead. The Sixteen Personality Factor Questionnaire (16PF) [e-book] The sage handbook of personality theory and assessment, [cited 2008 Jul 5] Available from: https://pdfs.semanticscholar. org/3ce1/2d90634b385bb0dfb25bdf0f4d192231e83a.pdf

21.Mel'nikov VD, Iampol'skij LT. Introduction to experimental psychology of personality. Moscow, 1985. (in Russian)

22.Faubert J. Professional athletes have extraordinary skills for rapidly learning complex and neutral dynamic visual scenes. Scientific Reports, 2013; 3. https://doi.org/10.1038/srep01154

23.Korobeynikov G, Mazmanian K, Korobeynikova L, Jagiello W. Diagnostics of psychophysiological states and motivation in elite athletes. Bratisl Lek Listy, 2011;112:637-43.

24.Tsos A, Hylchuk Y, Andreichuk O, Pantik V, Tsymbaliuk S Physical and Mental Health Components Condition in The Life Quality of Students Who Regularly Practice Kickboxing and Yoga, Phys Activ Rev, 2017;5: 37-43. https://doi.org/10.16926/par.2017.05.06

25.Vygotskij LS. The problem of the development of higher mental functions. In: Developmental psychology as a cultural phenomenon. Moscow-Voronezh; 1996; 1: P. 219261. (in Russian)

26.Zeigarnik BV. Kurt Lewin and Soviet psychology. Journal of Social, 1984; 40: 193-200.

\section{Information about the authors:}

Kolosov A.B.; PhD., http://orcid.org/0000-0001-6834-940X; andriy_kolosov@i.ua; Laboratory of Current and Operative Control of National Teams' Performance, State Scientific Research Institute of Physical Culture and Sports; 19, Stolychne Shose, 03131, Kyiv, Ukraine.

Volianiuk N.Yu.; (Corresponding author); Professor, Dr.; http://orcid.org/0000-0001-6945-5984; n.volianiuk1@gmail.com; Department of Psychology and Pedagogy, National Technical University of Ukraine «lgor Sikorsky Kyiv Polytechnic Institute»; 37 Peremogy Avenue, 03056 Kyiv, Ukraine.

Lozhkin G.V.; Professor, Dr.; http://orcid.org/ 0000-0002-6705-9344; lozhkin35@gmail.com; Department of Psychology and Pedagogy, National Technical University of Ukraine «lgor Sikorsky Kyiv Polytechnic Institute»; 37 Peremogy Avenue, 03056 Kyiv, Ukraine.

Buniak N.A.; Professor, Dr.; http://orcid.org/0000-0002-1129-6623; bunjak_n@tntu.edu.ua; Department of Psychology in production Ternopil Ivan Puluj National Technical University, Ruska str., 56, 46000 Ternopil, Ukraine.

Osodlo V.I.; Professor, Dr.; http://orcid.org/0000-0003-2893-47214; v.osyodlo@gmail.com; Social Studies Institute, National Defence University of Ukraine named after Ivan Cherniakhovskyi, Povitroflotskyi Pr., 28, 03049 Kyiv, Ukraine.

Cite this article as:

Kolosov AB, Volianiuk NYu, Lozhkin GV, Buniak NA, Osodlo VI. Personal determinants of mental reliability of an athlete. Pedagogics, psychology, medical-biological problems of physical training and sports, 2019;23(2):83-89. https://doi.org/10.15561/18189172.2019.0205

The electronic version of this article is the complete one and can be found online at: https://www.sportpedagogy.org.ua/index.php/PPS/issue/archive

This is an Open Access article distributed under the terms of the Creative Commons Attribution License, which permits unrestricted use, distribution, and reproduction in any medium, provided the original work is properly cited (http://creativecommons.org/licenses/by/4.0/deed.en).

Received: 10.03 .2019

Accepted: 15.04.2019; Published: 30.04.2019 